\title{
Improved Degradation Efficiency of Levofloxacin by a Self-Powered Electrochemical System with Pulsed Direct-Current
}

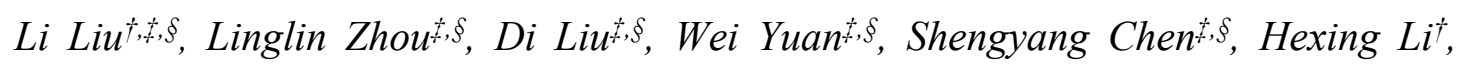

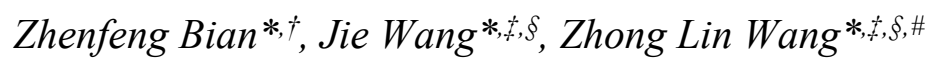

${ }^{\dagger}$ Education Ministry Key and International Joint Lab of Resource Chemistry and Shanghai Key Lab of Rare Earth Functional Materials, Shanghai Normal University, Shanghai 200234, China

¥Beijing Institute of Nanoenergy and Nanosystems, Chinese Academy of Sciences, Beijing 100083, China

${ }^{\S}$ College of Nanoscience and Technology, University of Chinese Academy of Sciences, Beijing 100049, China

\#School of Materials Science and Engineering, Georgia Institute of Technology, Atlanta, GA 30332, USA

\section{Corresponding Authors}

*E-mail: bianzhenfeng@shnu.edu.cn

*E-mail:wangjie@binn.cas.cn

*E-mail: zhong.wang@mse.gatech.edu

\section{Author Contributions}

Li Liu, Linglin Zhou, Di Liu contributed equally to this work. 
(a)

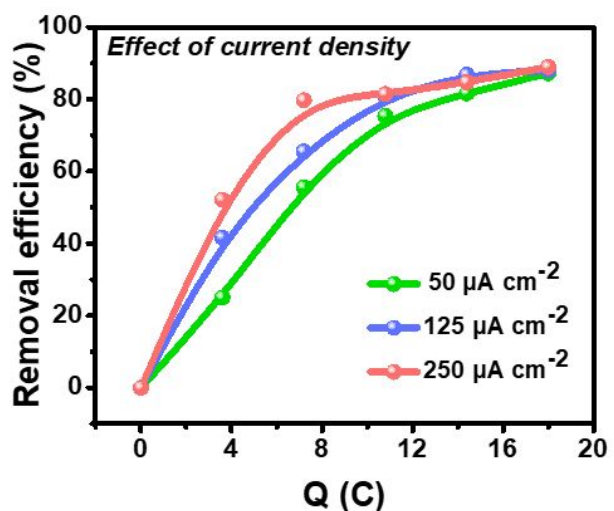

(c)

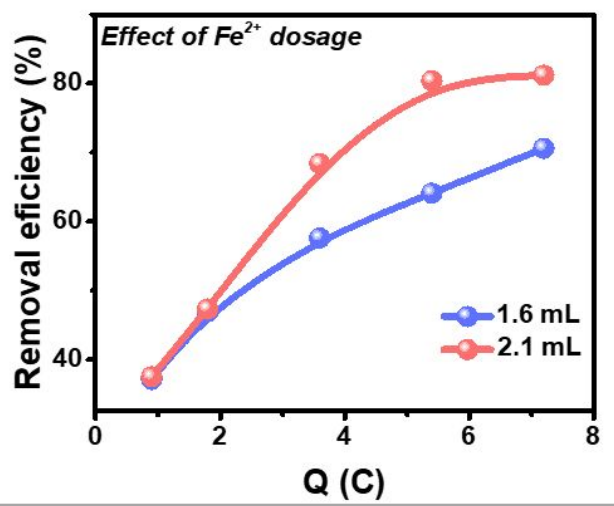

(b)

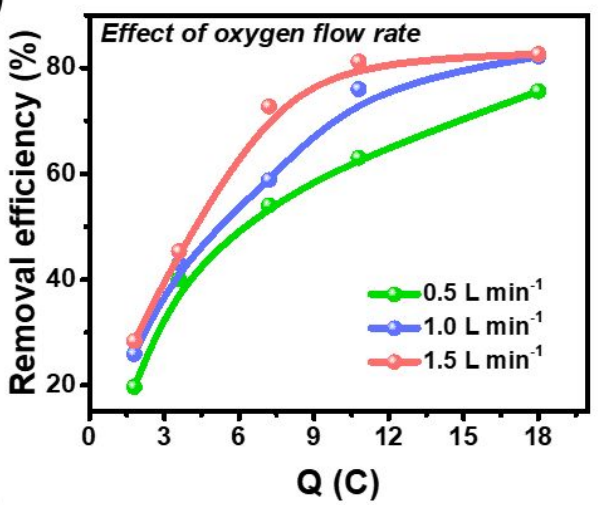

(d)

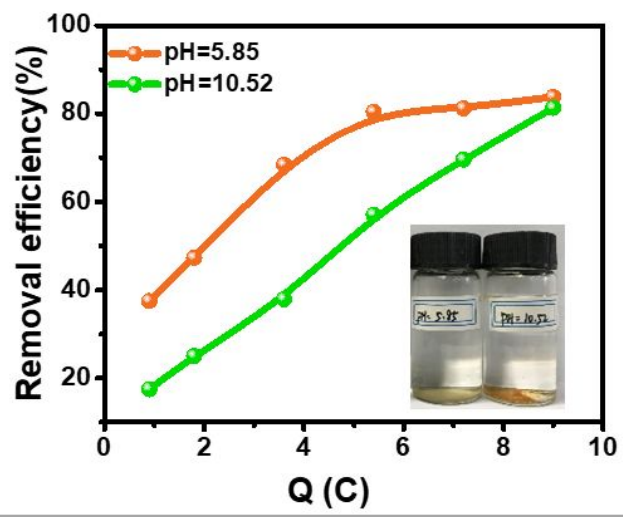

Figure S1. The influence of various factors on the removal efficiency of OFL driven by electrochemical workstation. (a) Effect of different current density. (b) Effect of different oxygen flow rate. (c) Effect of different $\mathrm{Fe}^{2+}$ dosage. (d) Removal efficiency of OFL at acid and alkaline conditions. 
(a)

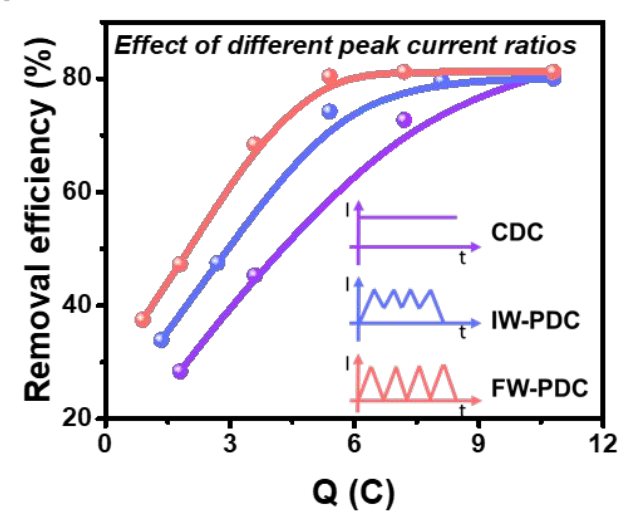

(b)

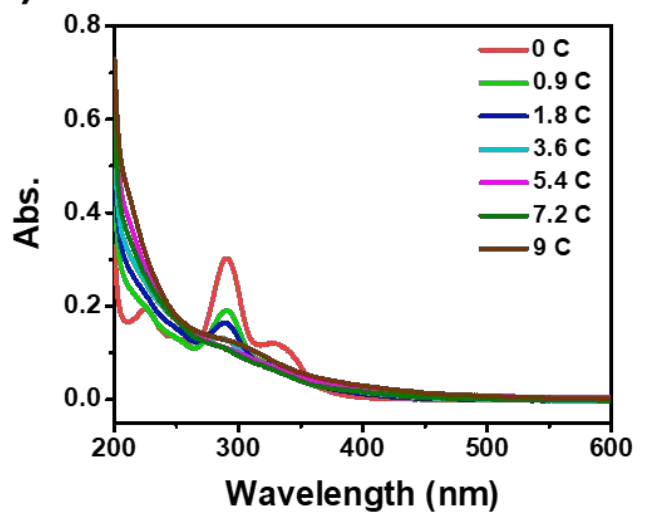

Figure S2. Effect of different current waveforms on OFL removal. (a) Effect of different current waveforms. (b) UV-Vis adsorption spectra of OFL removal for the different energy consumption by using FW-PDC. 
(a)

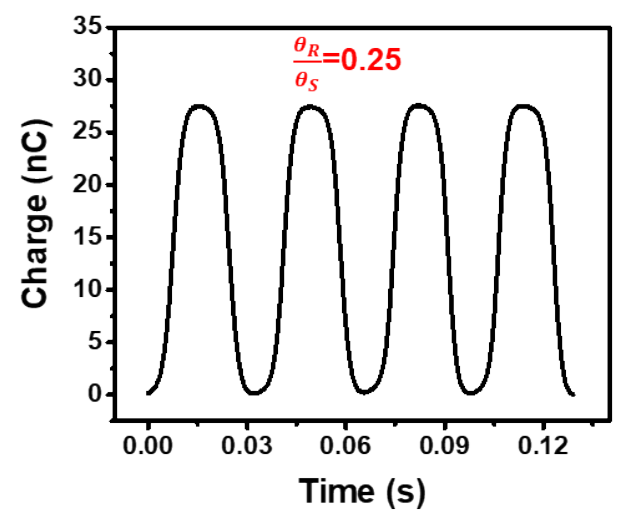

(c)

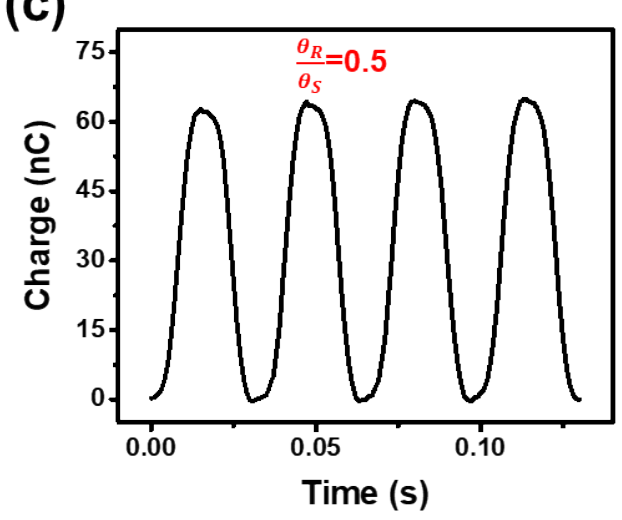

(b)

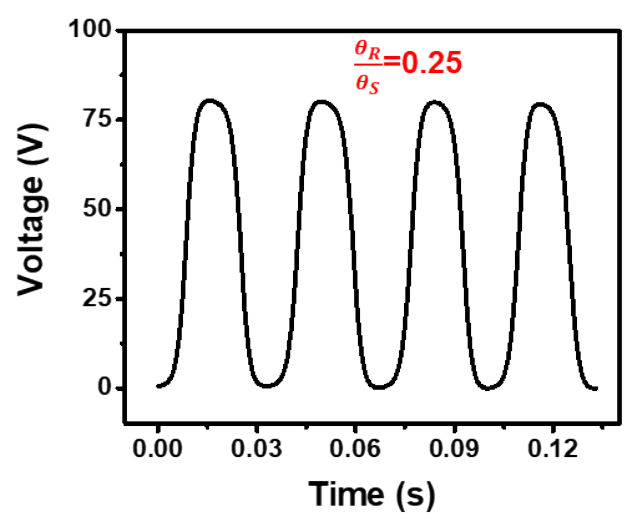

(d)



Figure S3. Electric output performances of R-TENG- $\theta_{R}$ with different ratios between $\theta_{R}$ and $\theta s$ at the rotation speed of $100 \mathrm{rpm}$. (a,b) A transferred charge and open-circuit voltage output performances of R-TENG-2.5 ${ }^{\circ}$ (c,d) A transferred charge and open-circuit voltage output performances of R-TENG-5 $5^{\circ}$ The diameter of R-TENG is $88 \mathrm{~mm}$. 
(a)



(b)

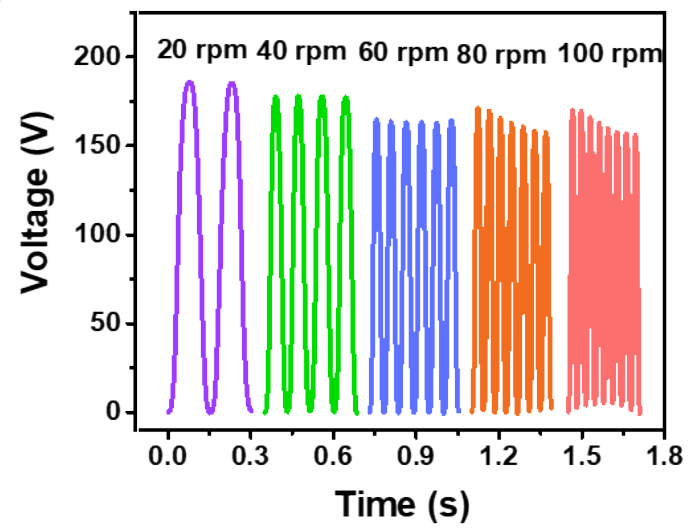

Figure S4. Electric output performances of R-TENG- $5^{\circ}$ with ratio between $\theta_{R}$ and $\theta_{S}$ of 0.5. (a) The transferred charge of R-TENG-5 ${ }^{\circ}$ at different speed. (b) The open-circuit voltage of the R-TENG- $5^{\circ}$ at different rotation speed. The diameter of $\mathrm{R}-\mathrm{TENG}-5^{\circ}$ is $88 \mathrm{~mm}$.

(a)

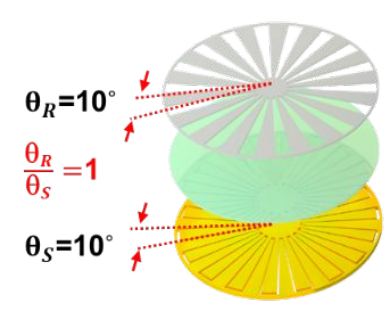

(b)

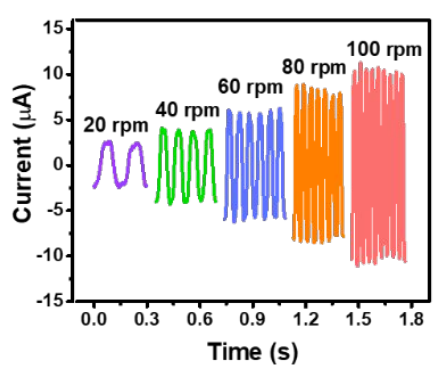

(c)

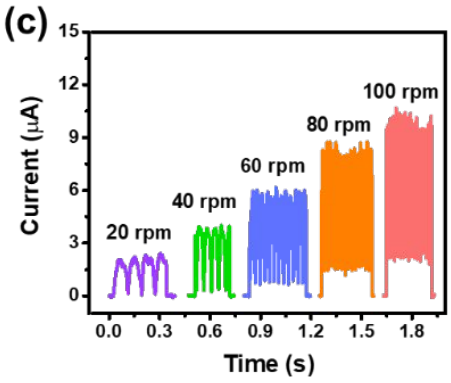

Figure S5. The output performances of R-TENG-10 $10^{\circ}$ with ratio between $\theta_{R}$ and $\theta_{S}$ of 1 at different rotation speed. (a) Schematic diagram of the R-TENG- $10^{\circ}$ structure with multiple rotating electrodes. (b) Short-circuit current output performances. (c) Rectified short-circuit current. The diameter of R-TENG- $10^{\circ}$ is $88 \mathrm{~mm}$. 
(a)

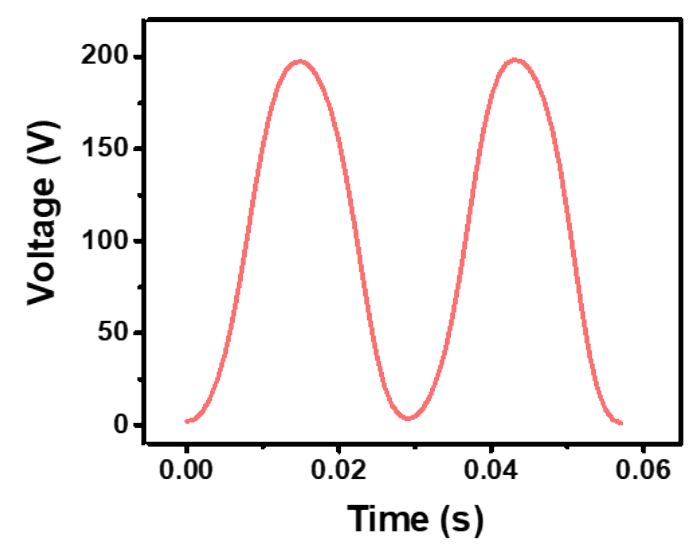

(b)

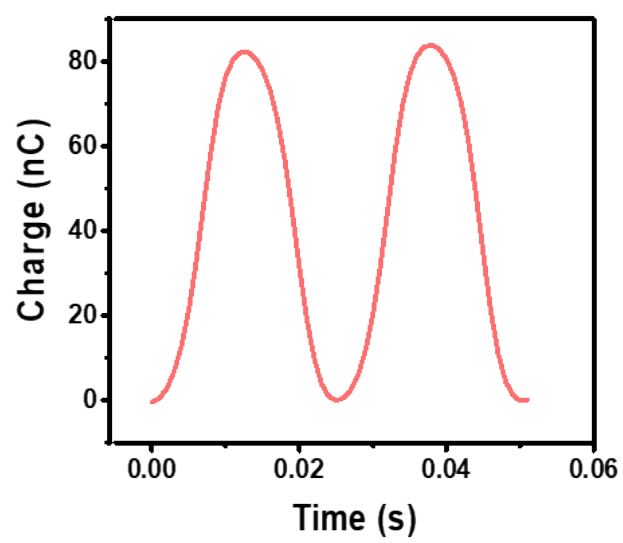

Figure S6. The electric output performances of the self-powered system based on R-TENG- $5^{\circ}$ driven by wind. (a) A voltage output performance. (b) The transferred charge output performance. The diameter of R-TENG-5 is $208 \mathrm{~mm}$. 